\title{
DEREGULATION AND QUALITY
}

\author{
Emmanuelle AURIOL ${ }^{1}$
}

July 1996

${ }^{1}$ GREMAQ/IDEI, University of Toulouse I, Place Anatole-France, 31042 Toulouse cedex, France; Tel: (33) 61-12-86-20; fax: (33) 61-12-86-37; email: auriol@gremaq.univ-tlse1.fr; I thank Jean-Jacques Laffont, Jean Tirole and two referees of this journal for their very helpful comments and advices. I am also very grateful to Richard Gilbert for his hospitality and to the Ministère des Affaires Etrangères (Lavoisier program) for financial support. 


\begin{abstract}
This paper analyses, within a static model, the effect of quality concern on optimal market structure. It focuses on cases where industry quality has public-good like features and is not contractible. It is shown that the introduction of competition raises a free-rider problem which depresses quality (the smaller producer free-rides on its competitor investment which as a result underinvests). To encourage the efficient producer to provide quality, the regulator diminishes the market-share of the opportunistic producer and chooses more often a monopoly. However when quality is verifiable the introduction of competition entails no welfare loss whether the regulator observes total quality outcome or individual contributions.
\end{abstract}

JEL classification: D43, D44, D82, L15, L43. 


\section{Introduction}

The quality of service in public utility industries has many dimensions. For example, the quality of electricity, gas, or telecommunication supply is defined not only by the physical specifications of the commodity (e.g., the voltage of electricity or the heating value of gas), but also by the reliability and frequency of the service, the safety of the technologies, and the expanse of client services (the clarity of the bills, the time of intervention in case of emergency, and so on). Some of these components are verifiable but many are not. In particular it may be very costly, if not impossible, to prove in court that electricity or telecommunication networks are built or run in an inefficient way, that the current purveyors of the industry underestimate the gain which would arise from the construction of new transmission facilities, or that the probability of a core meltdown at a nuclear plant fails to meet the social optimum. Since it is hard to include quality in a formal contract, regulatory agencies have devoted more attention to quantity and price control than to the promulgation of unenforceable standards. The responsibility for maintaining the quality of the service remains to a large extent with the supplying companies. ${ }^{1}$

There is an extensive literature on the provision of quality by unregulated firms (for a nice survey, see Tirole (1988) chapter 2). Although this seems to be an important issue to explore in a regulated environment, it has been largely overlooked in the past. $^{2}$ The aim of this paper is to study how the introduction of competition (i.e, a duopoly structure) in a traditionally regulated monopoly affects the provision of quality when some aspects of the transaction are not contractible and when quality has public-good like features. It contributes to a new literature

\footnotetext{
${ }^{1}$ When quality is verifiable it can be included in a contract exactly like a quantity (for the regulation of a multiproduct firm see, for example, Sappington (1983), Laffont-Tirole (1990)).

${ }^{2}$ To our knowledge, there are very few works on this topic. Lewis-Sappington (1991) compares the optimal regulation of a monopoly when the quality is verifiable with that when it is not. Laffont-Tirole (1993) focus on the link between quality provision and power of incentive schemes.
} 
on endogenous market structure in contracting environment which assess the impact of asymmetric cost information on optimal regulatory policy and auction design. Along this line Anton-Yao (1989,1992), Anton (1994) examine split-award auctions in procurement with respect to quantity, Auriol-Laffont (1993), Auriol (1996-a, 1996-b) optimal auction with variable quantity under various cost structures, Dana-Spier (1994) study mechanism design with the discrete award choices of a duopoly, a monopoly or government self production, Wolinsky (1994), AntonGertler (1994) and McGuire-Riordan (1995) focus on models of spatial duopoly. The main result of this literature is that asymmetric information favors the monopoly structure -sole sourcing- when the market structure decision -the award of market shares- follows cost revelation by firms (ex post framework). The intuition for this result is the following. Under asymmetric information firms get informational rent that increases the final cost of acquisition. To lower this extra cost it is optimal to distort the market structure decision in favor of the monopoly because under this new contract term a firm that claims high-cost faces reduced probability of producing. This perspective lowers firms' incentive for inflating cost report. It is to note that this pro monopoly bias can be reversed in an ex ante framework. When the market structure decision precedes the contracting stage Auriol-Laffont (1993), Auriol (1993) and Riordan (1994) hence shown that asymmetric information often favors duopolistic structure. ${ }^{3}$

In this paper we study the impact of partial deregulation on the provision of quality. Since the reform waves of the seventies and eighties introduced competition in public utilities, transportation and communication industries, it seems important to analyze the impact of these transformations on sectors once asserted to be natural monopolies. The reforms anticipated the fact that intensified rivalry would result in greater

\footnotetext{
${ }^{3}$ This is in particular the case when the price elasticity of the demand is low. When the principal has established a single producer at the pre-contracting phase, the monopoly power of this firm is ex post (i.e. at the time of contracting) very high if quantity is not flexible. To prevent ex post extortion by the monopoly it is optimal to choose more often a duopoly.
} 
efficiency, reduced cost and price, and increased quality and product variety. But we wonder whether, depending on the industry characteristics, some of this goals are not inherently conflicting. In particular in industries involving fixed connections between suppliers and customers (e.g., gas, water, train, telephone, or electricity) the competition has to take place through a single network because it is inefficient to duplicate transmission facilities. The interconnection - which is a preliminary to competition- yields economies of scale but it also complicates operations. It ties the different producers into a system where each individual action has a repercussion on every other member of the net. This physical unity is what differentiates interconnected systems from other systems of distribution of goods and services. We refer to this "link-together" property as the interconnected system effect. It is at play in most network industries, in particular in the natural gas industry, where customers get the average quality of the gas in the pipelines at the time of their purchase, in the telephone industry, where congestion phenomenon and incidents affect blindly all users, and in the electric industry: "All components of an electric power system are physically connected, and all can be dramatically affected by events elsewhere in the system. [...] The failure of a single AC-DC converter in a Florida Power and Light Co. nuclear plant in December 1982, for instance, triggered loss of power to 556,000 customers from the Georgia border to the Florida Keys. [...] A modern power system is in fact one large machine." Joskow-Schmalensee (1985).

This paper is an attempt to take the complexity and the multidimensionality of public utilities services into account. ${ }^{4}$ In particular it studies how the introduction of competition affects quality -reliabilityprovision when quality has public-good like features. It is shown to depress it. When the market is split between two firms, the larger supplier is the only one that provides quality while its smaller competitor free-rides on the investment through the network. Since the producer

\footnotetext{
${ }^{4}$ They can be decomposed into the supply of a commodity, a level of reliability and a range of customers services options.
} 
that pays for the reliability of the system cannot fully internalize the gains of doing so, it underinvests. To cushion this problem, the marketsharing rule has to be distorted in favor of the larger supplier. This distortion adds to the incomplete information distortion in such a way that the monopoly structure is chosen more often. That is, reliability concern militates for monopolistic structure. It reduces the diversity of the service options available to consumers, but also raises quality. However when the aggregate outcome in quality is verifiable, the regulator is able to take advantage of the competition without giving up quality: she unbundles the quality provision activity from the production. In this case the introduction of competition entails no welfare loss. This result is true whether the regulator observes the total quality outcome or individual suppliers' contributions. ${ }^{5}$

The paper is organized as follows: Section 2 presents the model and the main assumptions. Section 3 characterizes the complete information solution. Section 4 presents the situation where the regulator has imperfect information about the firms' marginal cost. Section 5 summarizes our results and offers some concluding remarks.

\section{The model}

We consider a regulation problem in which a commodity can be supplied through a common network by two firms, firm $a$ and firm $b$. Let $q^{i}(\geq 0)$ denote the quantity produces by firm $i(=a, b)$, and $v^{i}(\geq 0)$ its quality contribution. The consumers' gross surplus function, which is common knowledge, is:

$$
\hat{\mathrm{S}}\left(q^{a}, v^{a}, q^{b}, v^{b}\right)=S\left(q^{a}+q^{b}, v^{a}+v^{b}\right)+q^{a} q^{b} .
$$

This function captures the basic features described in the introduc-

\footnotetext{
${ }^{5}$ This is consistent with Picard-Rey (1990) and McAfee-McMillan (1991) result on team models with both moral hazard and adverse selection (these authors shown that it makes no difference whether the principal observes the outcome of the team or individual contributions).
} 
tion. To see how it can be generated consider the following simple horizontal differentiation model. A continuum of consumers uniformly distributed over the unit interval have an inelastic demand for one unit of the good (the market size is normalized to $q=1$ ). They derive the same gross utility $s(v)$ for consuming one unit of the good at a quality $v$ (uniform due to interconnection), but then incur a "transportation" $\operatorname{cost} \delta$, that can be interpreted as a location in the space of good characteristics, proportional to their "distance" to the supplier. Their utility is: $s(v)-\delta$. The suppliers are exogenously located, when active, at the extreme points. With a single producer, lets say in 0 , the consumers gross surplus is: $S(q, v)=\int_{0}^{q} s(v)-\delta d \delta=s(v)-\frac{q^{2}}{2}$. With a duopoly (one firm in 0 the other in 1) with market share $q^{a}$ for firm $a$ and $q^{b}$ for firm $b\left(q^{a}+q^{b}=q\right)$, the aggregate gross surplus becomes: $S\left(q^{a}, q^{b}, v\right)=\int_{0}^{q^{a}} s(v)-\delta d \delta+\int_{0}^{q^{b}} s(v)-\delta d \delta=S(q, v)+q^{a} q^{b}$.

The interpretation of this function is the following. Due to the interconnection, the individual contributions mix and become indistinguishable to consumers (as it is the case in telecommunication, gas or electricity industry when several producers operate on the same network): the surplus depends upon the total output available in the economy, $q=q^{a}+q^{b}$, and upon the firms' aggregated quality contributions, $v=v^{a}+v^{b}{ }^{6}$ However along with the commodity goes a bundle of services which differentiate horizontally the producers (such as contract options, technical assistance, qualification of the staff, convenience of the offices, and so on). The consumers, who have varied tastes and needs, value the diversity. Their gross surplus function increases with the duopoly since it provides the greater variety of service. This diversity effect is captured by $q^{a} q^{b} \geq 0$. It implies that the consumers gross surplus is maximum for $q^{a}=q^{b}$. With a monopoly the menu of services shrinks, there is a loss in welfare. The surplus becomes $S\left(q^{i}, v^{i}\right)$.

We denote by $H(q, v)=S_{q}(q, v) \geq 0$ the partial derivative of $S(q, v)$

\footnotetext{
${ }^{6}$ We consider the sum but we could equivalently consider the mean (or any increasing function of aggregated quality satisfying the gross surplus concavity requirement).
} 
with respect to $q$. The inverse demand function for "good" $i$ can be written:

$$
P^{i}\left(q^{a}, q^{b}, v\right)=H(q, v)+q^{j} \quad i=a, b, j \neq i, .
$$

When the market structure is a monopoly with firm $i$ the inverse demand function becomes $P\left(q^{i}, v^{i}\right)=H\left(q^{i}, v^{i}\right) .{ }^{7}$ We assume that the consumers' gross surplus function and the inverse demand function for the good $i(=a, b)$ are increasing in $v$ and concave in $\left(q^{a}, q^{b}, v\right)$. A sufficient condition for this to happen is

A1

$$
\begin{aligned}
& S(q, v)+\frac{q^{2}}{2} \text { is increasing and strictly concave in }(q, v) ; \\
& H(q, v) \text { is increasing in } v \text { and strictly concave in }(q, v) .
\end{aligned}
$$

Moreover, we assume that the net surplus function $S(q, v)+q^{a} q^{b}-$ $P^{a}\left(q^{a}, q^{b}, v\right) q^{a}-P^{b}\left(q^{a}, q^{b}, v\right) q^{b}$, is increasing with the quality. That is ${ }^{8}$

$$
S_{v}(q, v)-H_{v}(q, v) q \geq 0 .
$$

This assumption implies that an improvement in quality increases the gross surplus of consumers more than it increases the gross profit of sales. The social gain of an improvement in quality exceeds the willingness to pay for it.

The producers face a two-part cost function: ${ }^{9}$

$$
C^{i}\left(\beta^{i}, q^{i}, v^{i}\right)=\beta^{i} q^{i}+v^{i} \quad i=a, b,
$$

where $\beta^{i}(\in[\beta, \bar{\beta}])$ is the efficiency parameter identifying the firm (a high $\beta^{i}$ corresponds to a high cost, i.e., an inefficient producer), $q^{i} \geq 0$ the quantity it produces and $v^{i} \geq 0$ its quality contribution. The cost function is linear with respect to quantity $q^{i}$. It features constant marginal cost as in Baron-Myerson (1982). On the other hand the quality

\footnotetext{
${ }^{7}$ We drop the index of the firm in $P(.,$.$) because when the market structure is a$ monopoly the aggregated consumers surplus does not depend on which firm serves the market but on how much it produces.

${ }^{8}$ The subscripts denote the partial derivative with respect to $v$.

${ }^{9}$ To simplify matters, we assume that there is no fixed cost to enter the market but this assumption is not essential.
} 
investment does not depend upon quantity (it is not variable). It can be interpreted as the amount of money dedicated to increase the capacity of the system above the level required to operate in normal conditions. The higher it is the smaller the probability of an incident. For instance in electricity the reliability of the system is a matter "of absorbing the dynamic power swings and remaining stable following a disturbance resulting in the loss of any single generating unit, transmission circuit or transformer" Spiewak-Weiss (1991). A reliable, stable, high-quality service is achieved through global overbuilding. It implies that it is difficult to distinguish between investments those designed to improve quality from those made for commercial purposes. The fixed costs cannot usually be attributed solely to one account. The quality investment is not verifiable.

We assume that the regulator is not able to observe the cost of the firms. The $\beta^{i}$ which are a private information of firms' manager, are assumed to be independently drawn from the same distribution. We denote by $F\left(\beta^{i}\right)$ the cumulative distribution function and by $f\left(\beta^{i}\right)$ the density function of $\beta^{i} \in[\underline{\beta}, \bar{\beta}]$. The functions $F($.$) and f($.$) are common$ knowledge. They satisfy the monotone hazard rate property (to avoid bunching):

Moreover we assume that all the agents are risk-neutral. The regulator maximizes a utilitarian social welfare function (the sum of net consumers surplus and firms profit), under the firms' individual rationality constraint taking into account that the social cost of public funds is $1+\lambda>1$ ( $\lambda$ is strictly positive because of the need to use distortive taxation to raise money). The firms reservation profit is normalized to zero.

Finally, the timing of the model and the instruments of each player are the following: the regulator proposes an auction mechanism with a set of contracts that specify in function of firms cost announcement 
market shares, transfer payments, and quality requirement when it is verifiable. The firms make their bid. The regulator sets the market structure (monopoly or duopoly), the quantities and the quality outcome (whenever it is verifiable) as defined by contracts. When quality is not verifiable, the firm or firms choose (simultaneously and independently) their contributions in quality. Depending on the quantities and on the aggregate quality outcome, consumers express an (inverse) demand for the services, $P^{i}\left(q^{a}, q^{b}, v\right) .{ }^{10}$ We assume that the regulator is not able to monitor this (inverse) demand (it is not verifiable). ${ }^{11}$ We must stress that the unverifiability of the (inverse) demand function is linked to the unverifiability of the quality. When quality is verifiable, the regulator monitors indirectly price while regulating quantity and quality (quantity while regulating price and quality).

\section{The case of complete information}

Let $W$ and $W_{M}^{i}$ denote the ex post utilitarian social welfare functions when the market structure is respectively a duopoly and a monopoly with firm $i=a, b\left(U^{i}\right.$ and $U_{M}^{i}$ are the corresponding firm $i$ 's profit):

$$
\begin{aligned}
& W=S(q, v)+q^{a} q^{b}+\lambda P^{a} q^{a}+\lambda P^{b} q^{b}-(1+\lambda)\left(\beta^{a} q^{a}+\beta^{b} q^{b}+v\right)-\lambda U^{a}-\lambda U^{b} \\
& W_{M}^{i}=S\left(q_{M}^{i}, v_{M}^{i}\right)+\lambda P\left(q_{M}^{i}, v_{M}^{i}\right) q_{M}^{i}-(1+\lambda)\left(\beta^{i} q_{M}^{i}+v_{M}^{i}\right)-\lambda U_{M}^{i} .
\end{aligned}
$$

The regulator maximizes under firms' individual rationality constraint the following problem with respect to market structure (monopoly

\footnotetext{
${ }^{10}$ Since under our assumptions, the demand functions admit for each level of quality an inverse demand function, it is a question of convenience that the regulator sets quantities rather than prices, and that consumers express inverse demand rather than demand.

${ }^{11}$ An alternative assumption would be that the demand is subject to some exogenous random shocks such that it is impossible to deduce from prices observation the quality level. Even if the firms are assumed to be risk-neutral, the regulator cannot exploit their neutrality (i.e., base the contract on ex-post sales) because they are protected by limited liability (which take the form of ex post rationality constraint).
} 
or duopoly), quantities, quality and producers' profit (or equivalently producers' transfer payment):

$$
\begin{array}{ll}
\operatorname{Max} & x^{D} W+x^{a} W_{M}^{a}+x^{b} W_{M}^{b} \\
\text { s.t. } & U^{i}=t^{i}+P^{i}\left(q^{a}, q^{b}, v\right) q^{i}-\beta^{i} q^{i}-v^{i} \geq 0 \\
& U_{M}^{i}=t_{M}^{i}+P\left(q_{M}^{i}, v_{M}^{i}\right) q_{M}^{i}-\beta^{i} q_{M}^{i}-v_{M}^{i} \geq 0
\end{array}
$$

Term $x^{D} \geq 0$ and $x^{i} \geq 0$ are the probabilities of choosing respectively a duopoly structure or a monopoly structure with firm $i(=a, b)$. We assume that it is worth producing even with high cost firms. That is, $x^{D}+x^{a}+x^{b}=1 \quad \forall \beta^{a}, \beta^{b} \in[\underline{\beta}, \bar{\beta}]$. Moreover, since the objective function is linear in $x^{D}, x^{a}$, and $x^{b}$, these probabilities are at the optimum either equal to 0 or 1 . We maximize independently $W, W_{M}^{a}$, and $W_{M}^{b}$ with respect to rent, quantities and quality. The optimal market structure is the one associated with the maximum of these three functions. To ensure that the functions $W$ and $W_{M}^{i}$ are concave we make the assumption A $4:{ }^{12}$

A4

$$
1 \leq \frac{H_{v}(q, v)}{-H_{q v}(q, v) q} \leq 2
$$

We derive the optimal solution. Under complete information $\left(\beta^{a}, \beta^{b}\right.$ known), the rents which are costly $(\lambda>0)$, are set to zero in each regimes: $U^{i *}=0$ and $U_{M}^{i}=0 \quad(i=a, b)$. Moreover let $q^{*}$ be solution of:

$$
H\left(q^{*}, v^{*}\right)-\frac{\beta^{a}+\beta^{b}}{2}=\frac{-\lambda}{1+\lambda} H_{q}\left(q^{*}, v^{*}\right) q^{*}-\frac{1+2 \lambda}{2(1+\lambda)} q^{*} .
$$

If $q^{*}>\frac{1+\lambda}{1+2 \lambda}\left|\beta^{b}-\beta^{a}\right|$, the optimal market structure is a duopoly with market share:

$$
\left\{\begin{array}{l}
q^{a *}\left(q^{*}, \beta^{a}, \beta^{b}\right)=\frac{1}{2} q^{*}+\frac{1+\lambda}{1+2 \lambda} \frac{\beta^{b}-\beta^{a}}{2} \\
q^{b *}\left(q^{*}, \beta^{a}, \beta^{b}\right)=\frac{1}{2} q^{*}+\frac{1+\lambda}{1+2 \lambda} \frac{\beta^{a}-\beta^{b}}{2} .
\end{array}\right.
$$

It is a monopoly with the more efficient producer, identified by $\min \left(\beta^{a}, \beta^{b}\right)$,

\footnotetext{
${ }^{12}$ The subscript $q$ (resp. $v$ ) denotes the partial derivative with respect to $q$ (resp. to $v)$.
} 
otherwise. The monopoly production level is solution of:

$$
H\left(q^{M *}, v^{M *}\right)-\min \left(\beta^{a}, \beta^{b}\right)=\frac{-\lambda}{1+\lambda} H_{q}\left(q^{M *}, v^{M *}\right) q^{M *} .
$$

The quality formula is the same in monopoly and duopoly regime. For an aggregated production level $q$, it is solution of:

$$
S_{v}(q, v)+\lambda H_{v}(q, v) q=1+\lambda .
$$

The optimal level of quality, which is characterized by the equality of the social marginal cost of providing it $1+\lambda$ with its social marginal benefit $S_{v}+\lambda H_{v} q^{h}$, is independent of the market structure. Indeed as the costs are linear and separable in $(\beta, q)$ and $v$, the social marginal cost of providing quality is the same whatever the market structure. Moreover, as the individual contributions in quality sum up in the surplus function, the social benefit of quality does not depend upon which firm provides it. It depends however on the total output $\left(v^{M *}=v\left(q^{M *}\right), v^{*}=v\left(q^{*}\right)\right)$.

In the duopoly case, because of the imperfect substitutability of the commodities, even with different incremental costs both firms produce. To maximize the consumer surplus, which increases with the diversity in service options, the production should be equally divided between the two producers. But for cost minimization, the more efficient firm alone should produce. The optimal sharing rule is the result of a tradeoff between these efficiency and diversity concerns. The more efficient producer receives a greater part of the market than its competitor (from equations (4) we have $\frac{1+\lambda}{1+2 \lambda} \frac{\beta^{i}-\beta^{j}}{2}>0$ when $\beta^{i}>\beta^{j}$ ). The gap between the production levels increases with the difference in the firms' cost parameters.

When the difference between the costs of the firms becomes large the duopoly structure is no longer attractive. The regulator chooses a monopoly held by the more efficient producer (the firm with the higher cost has a zero probability of being chosen as monopoly: $x_{M}^{i}\left(\beta^{a}, \beta^{b}\right)=0$ if $\left.\beta^{i}=\max \left(\beta^{a}, \beta^{b}\right)\right)$. Dividing equation (5) by $H_{q}\left(q^{M *}, v^{M *}\right) q^{M *}$, we see that the monopoly pricing formula follows the Ramsey rule (the lerner 
index is equal to the inverse of the price elasticity time $\frac{\lambda}{1+\lambda}$ ). Moreover it is to note that the production level of the firm in a monopoly position is independent of its competitor cost.

Comparing the monopoly and the duopoly welfare at their first best levels, we characterize the optimal market structure decision. It depends upon the relative forces of the efficiency and diversity concerns (the proof is in appendix). Let

$$
\Delta^{*}(\beta)=\frac{1+2 \lambda}{1+\lambda} q^{M *}(\beta)
$$

Proposition 1 If the difference between the cost parameters $\left|\beta^{b}-\beta^{a}\right|$, is greater than $\Delta^{*}\left(\min \left(\beta^{a}, \beta^{b}\right)\right)$, the monopoly structure is optimal under complete information; otherwise the duopoly structure is preferred.

Accordingly the duopoly structure becomes less attractive when one of the firms is much more efficient than the other $\left(\left|\beta^{b}-\beta^{a}\right|\right.$ is large) or when the market is small (it is easy to check that $\frac{d \Delta^{*}}{d q^{M *}} \geq 0$ ).

\section{The cases of asymmetric information}

We now consider the situation in which firms have private information about their cost characteristic. By using the revelation principle we restrict the analysis to direct revelation mechanisms. The regulator extracts the producers' private information through incentive compatible mechanisms. We focus on truthful (subgame perfect) Bayesian Nash equilibria.

\subsection{Individual quality contributions are verifiable}

In this section, which describes a benchmark case, we assume that the quality provided by each firm is verifiable and can be contracted upon. The principal faces a pure adverse selection problem; she adds firms' incentive compatibility constraints to the program. 
Let $U^{i}\left(\beta^{i}\right)=E_{\beta^{j}} U^{i}\left(\beta^{i}, \beta^{j} / \beta^{i}\right)$ denote the firm's expected utility when telling the truth. From the envelope theorem, first and second order incentive compatibility constraints are: $\left(\right.$ IC1) $\dot{U}^{i}\left(\beta^{i}\right)=-$ $E_{\beta^{j}}\left[x^{D}\left(\beta^{i}, \beta^{j}\right) q^{i}\left(\beta^{i}, \beta^{j}\right)+x^{i}\left(\beta^{i}, \beta^{j}\right) q_{M}^{i}\left(\beta^{i}, \beta^{j}\right)\right] ;$

$(I C 2) \frac{\partial}{\partial \beta^{i}} E_{\beta^{j}}\left[x^{D}\left(\beta^{i}, \beta^{j}\right) q^{i}\left(\beta^{i}, \beta^{j}\right)+x^{i}\left(\beta^{i}, \beta^{j}\right) q_{M}^{i}\left(\beta^{i}, \beta^{j}\right)\right] \leq 0\left(\forall \beta^{i} \in\right.$ $[\underline{\beta}, \bar{\beta}], i=a, b, j \neq i)$. As the expected rents are socially costly $(\lambda>0)$ and decreasing in $\beta^{i}$ (from $(I C 1)$ we have $\dot{U}^{i}\left(\beta^{i}\right) \leq 0$ ), the individual rationality constraints (to be satisfied ex post due to limited liability) $U_{M}^{i}\left(\beta^{i}, \beta^{j}\right) \geq 0$ and $U^{i}\left(\beta^{i}, \beta^{j}\right) \geq 0 \forall \beta^{i} \in[\underline{\beta}, \bar{\beta}]$ are binding at $\beta^{i}=\bar{\beta}$. That is $(I R): U_{M}^{i}\left(\bar{\beta}, \beta^{j}\right)=0, U^{i}\left(\bar{\beta}, \beta^{j}\right)=0$. Integrating $(I C 1)$ with respect to (IR) yields: $E_{\beta^{i}} U^{i}\left(\beta^{i}\right)=\int_{\underline{\beta}}^{\bar{\beta}} \int_{\underline{\beta}}^{\bar{\beta}}\left[x^{D} q^{i}+x^{i} q_{M}^{i}\right] \frac{F\left(\beta^{i}\right)}{f\left(\beta^{i}\right)} d F\left(\beta^{j}\right) d F\left(\beta^{i}\right)$. Substituting this expression into $W$ equation (1) and $W_{M}^{i}$ equation (2), the principal solves with respect to market structure, quantity and quality:

$$
\begin{gathered}
\operatorname{Max} \int_{\underline{\beta}}^{\bar{\beta}} \int_{\underline{\beta}}^{\bar{\beta}}\left\{x^{D} W+x^{a} W_{M}^{a}+x^{b} W_{M}^{b}\right\} d F\left(\beta^{a}\right) d F\left(\beta^{b}\right) \\
\text { s.t. } \quad(I C 2) .
\end{gathered}
$$

As under complete information, the regulator's maximization problem is linear in $x^{D}$ and $x^{i}(i=a, b)$. She maximizes independently $W$ and $W_{M}^{i}$ with respect to quantity and quality. The optimal market structure is the one associated with the maximum of the three functions. Appendix 2 shows that $(I C 2)$ constraints are met by the following solution.

Let $q^{a *}, q^{b *}$ be defined equation (4), and let $q$ be solution of:

$$
H(q, v)-\frac{\beta^{a}+\beta^{b}}{2}=\frac{-\lambda}{1+\lambda} H_{q}(q, v) q-\frac{1+2 \lambda}{2(1+\lambda)} q+\frac{\lambda}{2(\lambda+1)}\left[\frac{F\left(\beta^{b}\right)}{f\left(\beta^{b}\right)}+\frac{F\left(\beta^{a}\right)}{f\left(\beta^{a}\right)}\right] .
$$

If $q>\frac{1+\lambda}{1+2 \lambda}\left|\beta^{b}-\beta^{a}\right|+\frac{\lambda}{1+2 \lambda}\left|\frac{F\left(\beta^{b}\right)}{f\left(\beta^{b}\right)}-\frac{F\left(\beta^{a}\right)}{f\left(\beta^{a}\right)}\right|$, the optimal market structure is a duopoly with market shares:

$$
\left\{\begin{array}{l}
q^{a}\left(q, \beta^{a}, \beta^{b}\right)=q^{a *}\left(q, \beta^{a}, \beta^{b}\right)+\frac{1}{2} \frac{\lambda}{1+2 \lambda}\left[\frac{F\left(\beta^{b}\right)}{f\left(\beta^{b}\right)}-\frac{F\left(\beta^{a}\right)}{f\left(\beta^{a}\right)}\right] \\
q^{b}\left(q, \beta^{a}, \beta^{b}\right)=q^{b *}\left(q, \beta^{a}, \beta^{b}\right)+\frac{1}{2} \frac{\lambda}{1+2 \lambda}\left[\frac{F\left(\beta^{a}\right)}{f\left(\beta^{a}\right)}-\frac{F\left(\beta^{b}\right)}{f\left(\beta^{b}\right)}\right] .
\end{array}\right.
$$


It is a monopoly associated with $\min \left(\beta^{a}, \beta^{b}\right)$ otherwise. The monopoly production level is solution of:

$$
H\left(q^{M}, v^{M}\right)-\min \left(\beta^{a}, \beta^{b}\right)=\frac{-\lambda}{\lambda+1} H_{q}\left(q^{M}, v^{M}\right) q^{M}+\frac{\lambda}{1+\lambda} \frac{F\left(\min \left(\beta^{a}, \beta^{b}\right)\right)}{f\left(\min \left(\beta^{a}, \beta^{b}\right)\right)} .
$$

The optimal quality formula is the same as under complete information. For the appropriated agregated levels of production it satisfies equation (6).

Protected by the incomplete information efficient firm can always pretend to be inefficient - an impossible claim to verify- and be reimbursed accordingly. It then secures for each unit produced the difference between its claim and its true cost: the informational rent. The rent is a function of the production levels required from high-cost producers, but it does not depend on the quality outcome. Rent extraction and provision of quality are hence disconnected. This result is due to the separability in $v$ and $(\beta, q)$ in the cost function. Likewise under complete information the optimal quality level depends upon the total quantity produced and not upon the particular market structure. However the quality outcome is lower under asymmetric information since the quantities are distorted for rent extraction.

In the duopoly case the regulator uses simultaneously the marketshare allocation and the total duopoly output to reduce the informational cost. She modifies the sharing rule in favor of the more efficient producer (term $\frac{F\left(\beta^{j}\right)}{f\left(\beta^{j}\right)}-\frac{F\left(\beta^{i}\right)}{f\left(\beta^{i}\right)}$ in equation (10) which is positive when $\beta^{i} \geq \beta^{j}$ ). This policy discourages the firms from overstating their cost since under the new contract term they receive shrunk market share when inflating their report. As the informational cost increases with the aggregate duopoly output, the regulator distorts (lowers) also the total production to reduce the firms' informational rent (term $\frac{\lambda}{1+\lambda} \frac{1}{2}\left[\frac{F\left(\beta^{b}\right)}{f\left(\beta^{b}\right)}+\frac{F\left(\beta^{a}\right)}{f\left(\beta^{a}\right)}\right]$ in equation (9), which implies that $q^{*}\left(\beta^{a}, \beta^{b}\right) \geq$ $\left.q\left(\beta^{a}, \beta^{b}\right) \forall \beta^{a}, \beta^{b} \in[\underline{\beta}, \bar{\beta}]^{2}\right)$.

When the difference between the firms' cost is large, the duopoly 
structure is no longer attractive. Likewise under complete information the market structure is a monopoly held by the more efficient supplier. The production level which is independent of its competitor cost, is also distorted to reduce the cost of the monopoly informational rent. However, this distortion is lower than in the duopoly case since the average informational cost is smaller $\left(\frac{F\left(\min \left(\beta^{a}, \beta^{b}\right)\right)}{f\left(\min \left(\beta^{a}, \beta^{b}\right)\right)} \leq \frac{1}{2}\left[\frac{F\left(\beta^{a}\right)}{f\left(\beta^{a}\right)}+\frac{F\left(\beta^{b}\right)}{f\left(\beta^{b}\right)}\right]\right)$.

Let us now compare the monopoly and duopoly welfare under incomplete information. The following proposition is shown by similar arguments as proposition 1 . Let

$$
\Delta(\beta)+\frac{\lambda}{1+\lambda}\left[\frac{F(\beta+\Delta(\beta))}{f(\beta+\Delta(\beta))}-\frac{F(\beta)}{f(\beta)}\right]=\frac{1+2 \lambda}{1+\lambda} q^{M}(\beta) .
$$

Proposition 2 If the difference between the cost parameter $\left|\beta^{b}-\beta^{a}\right|$ is greater than $\Delta\left(\min \left(\beta^{a}, \beta^{b}\right)\right)$, the monopoly structure is optimal under incomplete information; otherwise the duopoly structure is preferred.

From assumption $\mathrm{A} 3$ it is easy to check that $\Delta(\beta)$, solution of equation (12), is positive. Moreover, we see from equations (7) and (12) that for a given quantity, the monopoly structure occurs more often under incomplete information than under complete information. This result generalizes. Comparing the first best solution to the incomplete information solution it is straightforward under assumptions A1 and A4 to show the following result.

Proposition 3 Asymmetric information distorts the optimal market structure towards monopoly. That is, $\Delta(\beta) \leq \Delta^{*}(\beta) \quad \forall \beta \in[\underline{\beta}, \bar{\beta}]$.

Asymmetric information increases production costs by charges proportional to the hazard rate. In order to lower these informational rents, the regulator distorts the market sharing rule in favor of the more efficient firm. This policy reduces the gain for an efficient producer of mimicking an inefficient one. The regulator distorts also the market structure decision in favor of the monopoly because by doing so she lowers the incentive for inflating cost report (a high-cost firm has a decreased 
probability of producing under incomplete information). Moreover, as the global production is reduced to lower the cost of rent extraction, all the distortions combine to lessen the benefit of a duopolistic structure. In other words, asymmetric information favors the monopoly structure when quality is verifiable which is consistent with the results on endogenous market structure under asymmetric information quoted in the introduction.

\subsection{Quality is unverifiable}

In this section we assume that the quality of the delivered product can be perfectly observed by the consumers and the supplier but is not verifiable to any third party. Moreover, since the inverse demand is not verifiable, the principal faces reduced opportunities for regulating the firms. She can choose the market structure, monitor transfers and quantities but not quality. She faces at the same time a problem of moral hazard and adverse selection.

4-2-1 First we focus on the subgame in quality. At a previous stage of the game the firms made their bids; according to these announcements market structure, production levels and transfers are set. Depending on the contract reached, the firm or firms then freely choose their outcome in quality. The quality outcome, $v^{m}\left(q_{i}^{m}\right)$, chosen by firm $i$ when it is in a monopoly position is solution of: ${ }^{13}$

$$
H_{v}\left(q_{i}^{m}, v^{m}\right) q_{i}^{m}-1=0 \quad i=a, b .
$$

Comparing this solution to the benchmark case where quality is verifiable (equations (6) and (13)), we get (the proof is in appendix):

Lemma 1 Other things being equal, the level of quality provided by the

\footnotetext{
${ }^{13}$ We assume that the monopoly problem in quality admits an interior solution. Under assumption A1, quantity and quality are gross complement (which here is equivalent to net complement), that is $S_{q v}(q, v)>0$. If they were gross/net substitute, $S_{q v}(q, v)=H_{v}(q, v)$ would be negative and the quality level provided by the monopoly would be at its minimum.
} 
monopoly is lower than its social optimum.

As we pointed out in Section 2, under assumption A2 an improvement in quality raises the gross consumer surplus more than it increases the gross profit of sales. As the monopoly is not able to capture the whole social gain generated by the quality outcome, it tends to underprovide quality. It does not internalize the non-monetary benefit of its investment.

If the market structure decided at stage 1 is a duopoly with allocated production $\left(q_{d}^{a}, q_{d}^{b}\right)$, the quality level provided by the firms does not coincide with that in the monopoly case. It satisfies proposition 4 (the proof is in appendix).

Proposition 4 When the market structure is a duopoly, there is only one firm that provides quality. It is the firm with the larger market share. The aggregate duopoly outcome, denoted $v^{d}\left(q_{d}^{a}, q_{d}^{b}\right)$, satisfies for $q^{d}=q_{d}^{a}+q_{d}^{b}$ :

$$
H_{v}\left(q^{d}, v^{d}\right) \max \left(q_{d}^{a}, q_{d}^{b}\right)-1=0 .
$$

Due to the interconnected system effect, the quality in the duopoly case depends upon the agregated contributions of the suppliers. Since they are not distinguishable to consumers, the provision of quality constitutes a team activity for the producers. The problem is that, as quantity and quality are complementary in their profit function, they disagree about what should be the collective outcome. The producer with the larger market share wants a higher level of quality than its smaller counterpart. This disagreement between the "team" members creates the free-rider problem. The smaller supplier cares less about quality than its opponent. It free-rides on the latter through the network, providing the authorized quantity at a minimum quality level.

Contrary to the verifiable case, the rule establishing quality as a function of quantities depends upon the market structure. Indeed, comparing equations (13) and (14) it is straightforward to show that for 
the same aggregate output (i.e., $q_{d}^{a}+q_{d}^{b}=q^{m}$ ), the monopoly and the duopoly do not yield the same quality level.

Proposition 5 The introduction of competition deteriorates the quality; for the same aggregate quantity, the quality outcome is always lower with a duopoly structure than with a monopoly.

The introduction of competition tends to degrade quality (reliability). The smaller producer which cares less about it, skimps on investment. It takes free advantage of each unit invested by its competitor and does not lose customers, while the latter is inadequately rewarded for the costs it incurs. This opportunist behavior discourages the larger supplier from investing properly. Therefore for the same aggregate output, the duopoly structure offers a less reliable service.

4-2-2 The regulator, having computed the quality level that she might expect as a function of the market structure and the quantities has to optimize on these variables. Since the cost functions are separable in $v$ and $(\beta, q)$, the quality level chosen by the firms at the second stage of the game depends only upon quantities, and not upon the cost parameters $\beta^{a}, \beta^{b}$ (see equations (13) and (14)). For this reason the incentive constraints, $(I C 1)$ and $(I C 2)$, remain unchanged from Section 4-1. The regulator maximizes with respect to market structure and quantity:

$$
\begin{gathered}
\operatorname{Max} \int_{\underline{\beta}}^{\bar{\beta}} \int_{\underline{\beta}}^{\bar{\beta}}\left\{x^{D} W+x^{a} W_{M}^{a}+x^{b} W_{M}^{b}\right\} d F\left(\beta^{a}\right) d F\left(\beta^{b}\right) \\
\text { s.t. } \quad v^{m}\left(q_{i}^{m}\right) \text { solution of (13) } \quad i=a, b \\
v^{d}\left(q_{d}^{a}, q_{d}^{b}\right) \text { solution of }(14) \\
(I C 2) .
\end{gathered}
$$

Before solving this problem, we establish the following preliminary result (the proof is in appendix). ${ }^{14}$

\footnotetext{
${ }^{14}$ Lemma 2 may seem obvious but it is not. In particular one can show that if the
} 
Lemma 2 The firm with the lower marginal cost always receives the larger part of the market.

It follows immediately from lemma 2 and proposition 5 that the more efficient firm alone provides quality since it always gets the larger market-share. This result enables us to solve the regulator maximization program (16). It is linear in $x^{D}, x^{a}$, and $x^{b}$; we maximize independently $W$ and $W_{M}^{i}$. In order to ensure the concavity of the problem, we make assumption A5:

A5

$$
H_{v}(q, v) q \text { and } H_{v}(q, v) \text { are concave in }(q, v) \text {. }
$$

Appendix 6 shows that under our assumptions the program (15) is concave and that $(I C 2)$ constraints are met by the following solution.

Let $q^{a}, q^{b}$ be defined equation (10), and $q^{d}$ be solution of:

$$
\begin{array}{r}
H\left(q^{d}, v^{d}\right)-\frac{\beta^{a}+\beta^{b}}{2}=\frac{-\lambda}{\lambda+1} H_{q}\left(q^{d}, v^{d}\right) q^{d}-\frac{1+2 \lambda}{2(1+\lambda)} q^{d}+\frac{\lambda}{2(\lambda+1)}\left[\frac{F\left(\beta^{b}\right)}{f\left(\beta^{b}\right)}+\frac{F\left(\beta^{a}\right)}{f\left(\beta^{a}\right)}\right] \\
(16) \\
-\frac{v_{q^{a}}^{d}+v_{q^{b}}^{d}}{2} \frac{S_{v}\left(q^{d}, v^{d}\right)+\lambda H_{v}\left(q^{d}, v^{d}\right) q^{d}-(1+\lambda)}{1+\lambda} \\
\text { If } q^{d} \geq \frac{1+\lambda}{1+2 \lambda}\left|\beta^{a}-\beta^{b}\right|+\frac{\lambda}{1+2 \lambda}\left|\frac{F\left(\beta^{a}\right)}{f\left(\beta^{a}\right)}-\frac{F\left(\beta^{b}\right)}{f\left(\beta^{b}\right)}\right|+\frac{H_{v}\left[S_{v}+\lambda H_{v} q^{d}-(1+\lambda)\right]}{-H_{v v} \max \left(q^{a}, q^{b}\right)(1+2 \lambda)},
\end{array}
$$
the optimal market structure is a duopoly with market shares:

$$
\left\{\begin{array}{l}
q_{d}^{a}=q^{a}\left(q^{d}, \beta^{a}, \beta^{b}\right)+\frac{v_{q^{a}}^{d}-v_{q^{b}}^{d}}{2} \frac{S_{v}+\lambda H_{v} q^{d}-(1+\lambda)}{1+2 \lambda} \\
q_{d}^{b}=q^{b}\left(q^{d}, \beta^{a}, \beta^{b}\right)+\frac{v_{q^{b}}^{d}-v_{q^{a}}^{d}}{2 \Theta} \frac{S_{v}+\lambda H_{v} q^{d}-(1+\lambda)}{1+2 \lambda}
\end{array}\right.
$$

It is the monopoly solution $q^{m}$ associated with $\min \left(\beta^{a}, \beta^{b}\right)$ otherwise. The monopoly production level is solution of:

$$
\begin{aligned}
H\left(q^{m}, v^{m}\right)-\min \left(\beta^{a}, \beta^{b}\right)=\frac{-\lambda}{\lambda+1} H_{q}\left(q^{m}, v^{m}\right) q^{m} & +\frac{\lambda}{\lambda+1} \frac{F\left(\min \left(\beta^{a}, \beta^{b}\right)\right)}{f\left(\min \left(\beta^{a}, \beta^{b}\right)\right)} \\
& -\left.v_{q^{m}}^{m} \frac{S_{v}+\lambda H_{v} q^{m}-(1+\lambda)}{1+\lambda}\right|_{\left(q^{m}, v^{m}\right)} .
\end{aligned}
$$

producers' costs are correlated or asymmetric (drawn independently from different distributions), even in a Baron-Myerson (1982) type of model, the less efficient firm may be selected to produce; for more on this see Demski-Sappington-Spiller (1987), Auriol (1993), Auriol (1996-a). 
The quality level provided by the monopoly satisfies equation (13) and the quality provided by the duopoly equation (14).

As we pointed out in lemma 1, the monopoly tends to underprovide quality because it does not take into account the non monetary benefit generated by its investment. The duopoly structure yields even less quality because the free-rider problem adds to this internalization problem.

In order to correct these distortions the regulator modifies the contracts. As she cannot directly control the quality, she has to use the production allocation rule to simultaneously extract the informational rents and enhance quality provision. In the duopoly case, she distorts the market-sharing rule in favor of the more efficient producer (term $\left(v_{q^{i}}^{d}-v_{q^{j}}^{d}\right) \frac{S_{v}+\lambda H_{v} q^{d}-(1+\lambda)}{2 \Theta(1+2 \lambda)}$ in equations (17) which is positive for $\left.\beta^{i}<\beta^{j}\right)$. This new distortion adds to the incomplete information distortion analyzed section 4-1. They both favor the more efficient firm which gets the larger part of the total production. By doing so the regulator simultaneously diminishes the gain of mimicking inefficient firm (producers face shrunk market-share when inflating cost report) and increases the interest in quality provision (quantity and quality are complement in profit function). The regulator distorts also the global production rule (she tends to expand total duopoly output) because by raising the quantity produced by each firm, in particular the quantity produced by the more efficient one, she raises also the quality. Everything else being equal, the aggregate duopoly output is larger when quality is not verifiable when that it is (term $\left(v_{q^{i}}^{d}+v_{q^{j}}^{d}\right) \frac{S_{v}+\lambda H_{v} q^{d}-(1+\lambda)}{2(1+2 \lambda)}$ in equation (16), which is positive under assumptions A2 and A4).

When the difference between the cost parameters is large the duopoly is no longer attractive. The optimal market structure is a monopoly held by the more efficient producer. In this case the free-rider problem disappears but the underprovision problem subsists. The regulator has to distort the production rule to solve it. She has a single instrument to achieve the conflicting goals of rent extraction and quality provision. 
To lower the cost of asymmetric information, the production must be decreased (term $\frac{\lambda}{\lambda+1} \frac{F\left(\min \left(\beta^{a}, \beta^{b}\right)\right)}{f\left(\min \left(\beta^{a}, \beta^{b}\right)\right)} \geq 0$ in equation (18)), but to raise quality it must be increased (term $-d v_{q^{m}}^{m}\left[S_{v}\left(q^{m}, v^{m}\right)+\lambda H_{v}\left(q^{m}, v^{m}\right) q^{m}-\right.$ $(1+\lambda)] \leq 0$ under A3, A4). The optimal monopoly production level depends upon the relative force of these two effects. It may be either smaller or bigger than in the verifiable quality case.

Let us now compare monopoly and duopoly welfare under incomplete information. Once noted that for a given agregated output, the duopoly quality outcome converges to the monopoly one when $\min \left(q_{d}^{a}, q_{d}^{b}\right)$ converge to 0 , proposition 6 is shown by similar arguments as proposition 1. Let

$$
\Delta_{d}+\frac{\lambda}{1+\lambda}\left[\frac{F\left(\beta+\Delta_{d}\right)}{f\left(\beta+\Delta_{d}\right)}-\frac{F(\beta)}{f(\beta)}\right]=\frac{1+2 \lambda}{1+\lambda} q^{m}(\beta)-\left.\frac{H_{v}\left[S_{v}+\lambda H_{v} q^{m}-(1+\lambda)\right]}{-(1+\lambda) H_{v v} q^{m}}\right|_{\left(q^{m}, v^{m}\right)} .
$$

Proposition 6 If the difference between the cost parameters, $\left|\beta^{a}-\beta^{b}\right|$, is greater than $\Delta_{d}\left(\min \left(\beta^{a}, \beta^{b}\right)\right)$, the monopoly structure is optimal under incomplete information; otherwise the duopoly structure is preferred.

Everything else being equal, the critical difference between the cost parameters is bigger when the quality is verifiable when that it is not (because of the term $\frac{H_{v}\left[S_{v}+\lambda H_{v} q^{m}-(1+\lambda)\right]}{-H_{v v} q^{m}}=\frac{H_{v}\left[S_{v}-H_{v} q^{m}\right]}{-H_{v v} q^{m}} \geq 0$ under assumptions A1, A2). This result generalizes. Indeed comparing the unverifiable quality case to the verifiable one, we get the following proposition (the proof is in appendix).

Proposition 7 Under our assumptions, the non verifiability of the quality distorts the optimal market structure towards monopoly. That is, $\Delta_{d}(\beta) \leq \Delta(\beta) \leq \Delta^{*}(\beta), \forall \beta \in[\beta, \bar{\beta}]$.

When the market is split between two firms, the larger supplier is the only one to contribute to the system reliability while its competitor free-rides on the investment. Discouraged by this behavior, the former underinvests. The regulator reacts to this problem by diminishing the 
market share of the opportunistic producer. This distortion combines with the asymmetrical information distortion. They both increase the gap between the two suppliers production which lowers the interest of duopolistic structure. ${ }^{15}$ As a result the regulator chooses more often a monopoly. This policy reduces the diversity in service options but, as a single firm bears all the consequences of its investment, it solves the free-rider problem and thus raises quality. Quality - reliability- concern favors the monopoly structure when it is not verifiable.

\subsection{The aggregate quality outcome is verifiable}

In this section we assume that the aggregate outcome in quality is verifiable, but that the individual contributions are not. Since the prices depend only upon aggregate quality outcome and individual production levels, as she regulates quantity outputs and total quality the principal indirectly controls the prices. She has more instruments to regulate the firms. In fact she is able to set contracts such that quality, quantity, and transfers coincide with the solution of the verifiable quality case. The following proposition is demonstrated in appendix.

Proposition 8 There is no welfare loss whether the regulator monitors the aggregate quality outcome or the individual contributions.

When the aggregate quality outcome is verifiable, the regulator is able to solve the free-rider problem posed by the coexistence of several suppliers without dissipating the benefits of competition. She replicates the incentives of a regulated monopoly by dissociating the provision of quality from production. For this purpose she sets one of the firms liable for the reliability of the whole system. This firm is rewarded in accordance with the cost of providing the required quality outcome. Its competitor, which is not involved in the quality provision, is allowed to

\footnotetext{
${ }^{15}$ The social benefit of enlarging service options is linked with the quantities each suppliers is authorized to sell; it is maximal when the producers' market share equalize.
} 
free-ride on the investment. By unbundling the regulated service, the principal is able to promote competition in the segment of the market which is likely to benefit from it while she maintains regulation on the part with natural monopoly characteristics. This dissociation process has been used to introduce competition in sectors like the natural gas industry (unbundling gas production from gas transportation) and the telephone industry (unbundling long distance services from local services). The argument advanced for dividing up the service is that it improves market performance by enhancing competition in several parts of it. However the economic efficiency of unbundling relies on how much, if any, economy of scale ensues when the unbundled activities are produced jointly. Here these economies are nonexistent because the cost functions are separable in the quality provision and in the production. It is likely to be as in the gas example where transportation and production are two distinct activities.

Figure 1 contrasts the optimal market structures under incomplete information when quality is and is not verifiable in the firms' cost space;

[Figure 1]

\section{Conclusion}

This paper has studied the introduction of (duopolistic) competition in industries where quality -reliability- has public-good like feature. Verifiability is a key element of the regulatory process. Indeed, when quality is unverifiable the introduction of competition raises a free-rider problem that cannot be solved. The problem arises because the firms form different quality ends when confronted to different market shares (quantity and quality are complement in their profit function). Yet, due to the interconnection, the provision of quality constitutes a team activity for them. As a result the smaller producer which cares less about quality free-rides on its opponent's investment. This opportunistic behavior discourages the efficient supplier from investing properly. The regulator 
responds to this problem by increasing the market share of the efficient firm and by choosing more often a monopoly. However when quality is verifiable (either at individual or aggregate level) the regulator is able to take advantage of competition without giving up quality. She dissociates (unbundles) the quality provision activity from the production activity. Since reliability, regularity, and safety are essential concerns in public utility industries, these results suggest that with verifiability problems, competition is more likely to yield net benefits under common carrier structures than under vertically integrated structures with obligated access.

From this point of view, the reform of the US electric power system does not correctly addresses the reliability issue. The Energy Policy Act (EPAct) enacted in October 1992, gives the Federal Energy Regulatory Commission expanded powers to mandate transmission access and reinforces the legitimacy of non-utility generators. These non-utility generators "have proved to be aggressive competitors; in recent years, they have accounted for more than half of new generation capacity brought on line in the United States" Watkiss-Smith (1993). Since their weight in the US power market is steadily increasing, their responsibility to the system operating reliability must be seriously addressed. Yet the EPAct, which preserves the current vertically integrated structure of the industry, deals only superficially with this issue. In light of our results we are afraid that the reform provokes in the future stability and reliability concerns. 


\section{APPENDIX 1 : proof of proposition 1}

Note that the monopoly problem is a particular case of the duopoly one: $W_{M}^{i}\left(q^{i}, v\right)=W\left(q^{i}, 0, v\right)$. Under assumption A1 $W$ being strictly concave in $\left(q^{a}, q^{b}, v\right)$, we deduce that the duopoly is the optimal market structure iff $\min \left(q^{a *}, q^{b *}\right)>0$; From equation (4) this is equivalent to $\left|\beta^{a}-\beta^{b}\right|<\frac{1+2 \lambda}{1+\lambda} q^{*}\left(\beta^{a}, \beta^{b}\right)$. Let $\Delta^{*}(\beta)=\frac{1+2 \lambda}{1+\lambda} q^{*}\left(\beta, \beta+\Delta^{*}\right)$ and substitute it into (3) yields: $q^{*}\left(\beta, \beta+\Delta^{*}\right)=q^{M *}(\beta)$; we deduce easily the result. $\diamond$

\section{APPENDIX $2:(I C 2)$ constraints}

Define $\psi(\beta)=\Delta(\beta)+\beta$, where $\Delta(\beta)$ is solution of equation (12). Differentiating $\psi(\beta)$ we get $\frac{d \psi(\beta)}{d \beta} \equiv \frac{(1+2 \lambda) H_{q}+\lambda H_{q q} q+(1+2 \lambda)}{(1+2 \lambda) H_{q}+\lambda H_{q q} q}>0$, under A1, A3. As $\psi(\beta)$ is strictly increasing in $\beta \in[\underline{\beta}, \bar{\beta}], \psi^{-1}(\beta)$, the inverse function of $\psi(\beta)$, exists and is also increasing in $\beta \in[\psi(\underline{\beta}), \psi(\bar{\beta})]$. We define $\Psi($.$) and \Psi^{-1}($.$) the prolongation of \psi($.$) and \psi^{-1}($.$) to \mathbb{R}$ :

$$
\begin{aligned}
& \Psi(\beta)= \begin{cases}\underline{\beta} \quad \text { if } \beta \leq \psi^{-1}(\underline{\beta}) \\
\psi(\beta) \text { if } \beta \in\left[\psi^{-1}(\underline{\beta}), \psi^{-1}(\bar{\beta})\right] \\
\bar{\beta} \quad \text { if } \beta \geq \psi^{-1}(\bar{\beta})\end{cases} \\
& \Psi^{-1}(\beta)= \begin{cases}\underline{\beta} & \text { if } \beta \leq \psi(\underline{\beta}) \\
\psi^{-1}(\beta) & \text { if } \beta \in[\psi(\bar{\beta}), \psi(\bar{\beta})] . \\
\bar{\beta} & \text { if } \beta \geq \psi(\bar{\beta})\end{cases}
\end{aligned}
$$

Proposition 2 implies: $x^{i}\left(\beta^{a}, \beta^{b}\right)=1 \Leftrightarrow \beta^{j} \geq \Psi\left(\beta^{i}\right), \quad x^{d}\left(\beta^{a}, \beta^{b}\right)=1 \Leftrightarrow$ $\Psi^{-1}\left(\beta^{i}\right) \leq \beta^{j} \leq \Psi\left(\beta^{i}\right)$. We deduce:

$E_{\beta^{j}}\left[x^{i} q^{M}+x^{D} q^{i}\right]=\left[1-F\left(\Psi\left(\beta^{i}\right)\right)\right] q^{M}\left(\beta^{i}\right)+\int_{\Psi^{-1}\left(\beta^{i}\right)}^{\Psi\left(\beta^{i}\right)} q^{i}\left(\beta^{a}, \beta^{b}\right) d F\left(\beta^{j}\right)$.

It is next easy to check the following conditions:

(i) $q^{i}\left(\beta^{i}, \Psi^{-1}\left(\beta^{i}\right)\right)=0$ if $\beta^{i} \in[\psi(\underline{\beta}), \psi(\bar{\beta})]$ and $\dot{\Psi}^{-1}\left(\beta^{i}\right)=0$ otherwise,

(ii) $q^{i}\left(\beta^{i}, \Psi\left(\beta^{i}\right)\right)=q_{M}^{i}\left(\beta^{i}\right)$ if $\beta^{i} \in\left[\psi^{-1}(\underline{\beta}), \psi^{-1}(\bar{\beta})\right]$ and $\dot{\Psi}\left(\beta^{i}\right)=0$ otherwise.

Differentiating (IC1) and simplifying with conditions (i) and (ii), yields: $\frac{\partial E_{\beta^{j}}\left(x^{D} q^{i}+x^{i} q^{M}\right)}{\partial \beta^{i}}=\left[1-F\left(\Psi\left(\beta^{i}\right)\right)\right] \frac{d q^{M}\left(\beta^{i}\right)}{d \beta^{i}}+\int_{\Psi^{-1}\left(\beta^{i}\right)}^{\Psi\left(\beta^{i}\right)} \frac{\partial q^{i}\left(\beta^{a}, \beta^{b}\right)}{\partial \beta^{i}} d F\left(\beta^{j}\right)$. Sufficient conditions for $(I C 2)$ are: $\frac{d q^{M}}{d \beta} \leq 0$ and $\frac{\partial q^{i}\left(\beta^{a}, \beta^{b}\right)}{\partial \beta^{i}} \leq 0$. Under 
$\mathrm{A} 1, \mathrm{~A} 4, \mathrm{~A} 3, \frac{d q^{M}}{d \beta}=\frac{\left[1+\lambda+\lambda \frac{d}{d \beta} \frac{F(\beta)}{f(\beta)}\right]\left[S_{v v}+\lambda H_{v v} q\right]}{\left[(1+2 \lambda) H_{q}+\lambda H_{q q} q\right]\left[S_{v v}+\lambda H_{v v} q\right]-\left[(1+\lambda) H_{v}+\lambda H_{v q} q\right]^{2}} \leq 0$, and from equation (10) $\frac{\partial q^{i}\left(\beta^{a}, \beta^{b}\right)}{\partial \beta^{i}} \leq 0$ as soon as $q$ defined equation (9) is decreasing in $\beta^{i}$. That is under A1, A3, A4:

$$
\frac{\partial q}{\partial \beta^{i}}=\frac{\frac{1}{2}\left[1+\lambda+\lambda \frac{d}{d \beta^{i}} \frac{F\left(\beta^{i}\right)}{f\left(\beta^{i}\right)}\right]\left[S_{v v}+\lambda H_{v v} q^{D}\right]}{\left[(1+2 \lambda) H_{q}+\lambda H_{q q} q+\frac{1+2 \lambda}{2}\right]\left[S_{v v}+\lambda H_{v v} q\right]-\left[(1+\lambda) H_{v}+\lambda H_{v q} q\right]^{2}} \leq 0 . \diamond
$$

\section{APPENDIX 3 : proof of lemma 1}

Let $\Phi(v)=\frac{1}{1+\lambda}\left[S_{v}(q, v)+\lambda H_{v}(q, v) q\right]$ and $\Pi(v)=H_{v}(q, v) q$. For a given $q$ the optimal quality level is solution of: $\Phi(v)=1$; the level chosen by the monopoly is solution of: $\Pi(v)=1$. Since under assumption A1 $\Phi(v)$ and $\Pi(v)$ are decreasing in $v$ and since under assumption A2 $\Phi(v) \geq \Pi(v) \forall v \geq 0$, we deduce easily the result. $\diamond$

\section{APPENDIX 4 : proof of proposition 4}

Firm $i$ solves with respect to $v^{i}: \operatorname{Max}\left[t^{i}\left(\tilde{\beta}^{i}, \tilde{\beta}^{j}\right)+P^{i}\left(q_{d}^{a}, q_{d}^{b}, v^{d}\right) q_{d}^{i}\left(\tilde{\beta}^{i}, \tilde{\beta}^{j}\right)-\right.$ $\left.v^{i}-\beta^{i} q_{d}^{i}\left(\tilde{\beta}^{i}, \tilde{\beta}^{j}\right)\right]$. We focus on Nash equilibria. The first order conditions are such that: $U_{v^{i}}^{i}=H_{v}\left(q^{d}, v^{d}\right) q_{d}^{i}-1=0(i=a, b)$. Except when the quantities $q_{d}^{a}$ and $q_{d}^{b}$ are equal, there is no interior solution to this duopoly quality problem. Let $v^{i+}$ be the level of aggregate quality which maximizes the firm $i$ profit $\left(v^{i+}\right.$ is such that $\left.H_{v}\left(q^{d}, v\right) q_{d}^{i}\left(\tilde{\beta}^{a}, \tilde{\beta}^{b}\right)=1\right)$, the firm $i$ best reponse function to firm $j$ is: $V^{i}\left(v^{j}\right)=v^{i+}-v^{j}$ if $v^{j} \leq v^{i+}$ and $V^{i}\left(v^{j}\right)=0$ otherwise. Note next that under assumptions A1 and A4, $\frac{d v^{i+}}{d q_{d}^{i}}=\frac{H_{v q} q_{d}^{i}+H_{v}}{-H_{v v} q_{d}^{i}} \geq 0$; that is, $q_{d}^{i}>q_{d}^{j} \Leftrightarrow v^{i+}>v^{j+}$. There exists a unique Nash equilibrium for this game. It is given by: $q_{d}^{i}>q_{d}^{j} \Rightarrow v_{d}^{i}=v^{i+}, v_{d}^{j}=0 . \diamond$

\section{APPENDIX 5 : proof of lemma 2}

Assume that $\beta^{b}>\beta^{a}$, and that at the optimum $q_{d}^{b}>q_{d}^{a}$. Consider next the following transformation: $q^{a+}=q_{d}^{a}+\left(q_{d}^{b}-q_{d}^{a}\right)$ and $q^{b+}=$ $q_{d}^{b}-\left(q_{d}^{b}-q_{d}^{a}\right)$, which preserves unchanged the total output $\left(q_{d}^{a}+q_{d}^{b}=q^{a+}+\right.$ $\left.q^{b+}\right)$ and the quality provided by the duopoly $\left(v^{d}\left(q^{a+}, q^{b+}\right)=v^{d}\left(q_{d}^{a}, q_{d}^{b}\right)\right)$. Comparing the value of the program at $\left(q_{d}^{a}, q_{d}^{b}\right)$ and $\left(q^{a+}, q^{b+}\right)$, we get: 
$W^{+}=W+\left(q_{d}^{b}-q_{d}^{a}\right)\left[(1+\lambda)\left(\beta^{b}-\beta^{a}\right)+\lambda\left(\frac{F\left(\beta^{b}\right)}{f\left(\beta^{b}\right)}-\frac{F\left(\beta^{a}\right)}{f\left(\beta^{a}\right)}\right)\right]>W$ under assumption A3; a contradiction. $\diamond$

\section{APPENDIX 6 : Optimality of the Solutions}

Concavity of the regulator's program: Note that $W^{M}\left(q, v^{m}(q)\right)=$ $W\left(q, 0, v^{d}(q, 0)\right)$. $W^{M}\left(q, v^{m}(q)\right)$ is concave in $q$ if $W\left(q^{a}, q^{b}, v^{d}\left(q^{a}, q^{b}\right)\right)$ is concave in $\left(q^{a}, q^{b}\right)$ which is true if $(a) W_{q^{i} q^{i}} \leq 0$ and (b) $W_{q^{a} q^{a}} W_{q^{b} q^{b}}-$ $\left[W_{q^{a} q^{b}}\right]^{2} \geq 0$. it is easy to check although tedious, that condition $(a)$ is satisfied under assumptions $\mathrm{A} 1, \mathrm{~A} 4, \mathrm{~A} 5$, and that condition $(b)$ is satisfied under assumptions A1, A2, A4, A5.

(IC2) constraints: (same proof as in appendix 2 but with $\psi(\beta)$ replaced by $\psi_{d}(\beta)=\Delta_{d}(\beta)+\beta$ which is a strictly decreasing function under assumptions A1, A3, A5). Sufficient conditions are $\frac{d q^{m}(\beta)}{d \beta}=$

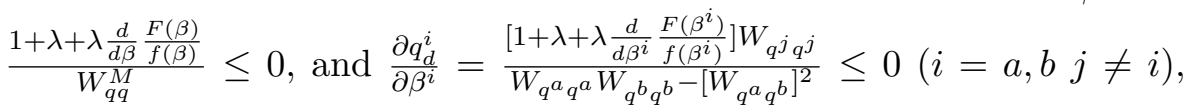
which are true under assumptions A1, A2, A3, A4, A5. $\diamond$

\section{APPENDIX 7 : Proof of Proposition 7}

Note that under A3 $x+\frac{\lambda}{1+\lambda}\left[\frac{F(\beta+x)}{f(\beta+x)}-\frac{F(\beta)}{f(\beta)}\right]$ is strictly increasing in $x$. A necessary and sufficient condition for proposition 7 is then: $q^{m}(\beta)-$ $q^{M}(\beta) \leq \frac{H_{v}\left[S_{v}+\lambda H_{v} q^{m}-(1+\lambda)\right]}{-(1+2 \lambda) H_{v v} q^{m}}$. Let $g(q)=v_{q}^{m} \frac{S_{v}\left(q, v^{m}\right)+\lambda H_{v}\left(q, v^{m}\right) q-(1+\lambda)}{1+2 \lambda}$, since $v_{q}^{m} \geq \frac{H_{v}\left(q, v^{m}\right)}{-H_{v v}\left(q, v^{m}\right) q}$ a sufficient condition is $q^{m}(\beta)-q^{M}(\beta) \leq g\left(q^{m}(\beta)\right)$. Define $\Phi(q)=\frac{(1+\lambda)\left[H\left(q, v^{m}\right)-\beta\right]+\lambda H_{q}\left(q, v^{m}\right) q-\lambda \frac{F(\beta)}{f(\beta)}}{1+2 \lambda}$, and let $Q^{M}(\beta)$ be solution of $\Phi\left(Q^{M}(\beta)\right)=0$. From lemma 1, $v(q)$ defined equation (6), is such that $v(q) \geq v^{m}(q) \forall q \geq 0$, and from A1, A4 $\frac{d q^{M}}{d v}=\frac{(1+\lambda) H_{v}+\lambda H_{q v} q}{-\left[(1+2 \lambda) H_{q}+\lambda H_{q q} q\right]} \geq$ 0 . We deduce that $q^{M}(\beta) \geq Q^{M}(\beta) \forall \beta \in[\beta, \bar{\beta}]$. It is thus sufficient that: $q^{m}(\beta)-Q^{M}(\beta) \leq g\left(q^{m}(\beta)\right)$. Since from equation (18) $q^{m}(\beta)$ is such as: $\Phi\left(q^{m}(\beta)\right)+g\left(q^{m}(\beta)\right)=0$, we rewrite this condition as $q^{m}(\beta)-Q^{M}(\beta) \leq \Phi\left(Q^{M}(\beta)\right)-\Phi\left(q^{m}(\beta)\right)$ which is true as soon as $\dot{\Phi}(q) \leq$ $-1 \forall q \geq 0$. This is equivalent to $\left[H_{q v} q+H_{v}\right]\left[(1+\lambda) H_{v}+\lambda H_{v q} q\right] \leq$ $H_{v v} q\left[(1+2 \lambda) H_{q}+\lambda H_{q q} q+1+2 \lambda\right]$. We deduce the result under assumptions A1, A2, A4. $\diamond$ 


\section{APPENDIX 8 : Proof of Proposition 8}

One of the two producers is set liable $(l)$ for the aggregate quality outcome. It is reimbursed for the cost incurred if quality coincides with equation (6). Otherwise, it suffers an infinite penalty. At stage 2 of the game, the best reponse function of the firm liable for the quality is $V^{l}\left(v^{n l}\right)=v\left(q^{a}+q^{b}\right)-v^{n l}$ if $v^{n l} \leq v\left(q^{a}+q^{b}\right)$, and $V^{l}\left(v^{n l}\right)=0$ otherwise, where $v\left(q^{a}+q^{b}\right)$, is the solution of equation (6). The best reponse function of its opponent $(n l)$ is $V^{n l}\left(v^{l}\right)=v^{d}\left(q^{a}, q^{b}\right)-v^{l}$ if $v^{l} \leq v^{d}\left(q^{a}, q^{b}\right)$, and $V^{n l}\left(v^{l}\right)=0$ otherwise. There exists a unique Nash equilibrium for this game. It is given by: $v^{l}=v\left(q^{a}+q^{b}\right), v^{n l}=0 . \diamond$ 


\section{REFERENCES}

ANTON, J. (1994), "Equilibrium Bids and Scale Economies in SplitAward Auctions", working paper, Duke University.

ANTON, J. and P. GERTLER (1994), "Regulation, Local Monopolies and Spatial Competition", mimeo.

ANTON, J. and D. YAO (1989), "Spit Award, Procurement and Innovation", RAND Journal of Economics, 20, 538-552.

ANTON, J. and D. YAO (1992), "Coordination in Spit-Award Auctions", Quartely Journal of Economics, 681-707.

AURIOL, E. and J.J. LAFFONT (1993), "Regulation by Duopoly", Journal of Economics and Management Strategy, 3, 507-533.

AURIOL, E. (1993), "Monopole ou Duopole : l'effet de Comparaison", Annales d'Economie et de Statistique, 31, 1-31.

AURIOL, E. (1996-a), "Une Note sur l'Effet d'Echantillonnage", Revue Economique.

AURIOL, E. (1996-b), "Delegation Under the Threat of Corruption in Procurement and Public Purshase", mimeo Toulouse.

BARON, D. and R. MYERSON (1982), "Regulating a Monopolist with Unknown Costs", Econometrica, 50, 911-930.

DANA, J. and K. SPIER (1994), "Designing a Private Industry : Government Auctions with Endogenous Market Structure", Journal of Public Economics, 53, 127-147.

GEGAX, D. and K. NOWOTNY (1993), "Competition and the Electric Utility Industry: An Evaluation", Yale Journal on Regulation, 1, 63-87.

JOSKOW, P. and R. SCHMALENSEE (1985), "Markets for Power", MIT Press.

LAFFONT, J.J. and J. TIROLE (1987), "Auctioning Incentives Con- 
tracts", Journal of Political Economy, 95.

LAFFONT, J.J. and J. TIROLE (1990), "The Regulation of Multiproduct Firms", Journal of Public Economics, 43, 1-36.

LAFFONT, J.J. and J. TIROLE (1993), "Regulation of Quality", $A$ Theory of Incentives in Procurement and Regulation, Chapter 4, MIT press.

LEWIS, T. and D. SAPPINGTON (1988), "Regulating a Monopolist with Unknown Demand", American Economic Review, 78, 986-998.

LEWIS, T. and D. SAPPINGTON (1991), "Incentives for Monitoring Quality", RAND Journal of Economics.

McAFEE, P. and J. McMILLAN (1991), "Optimal Contracts for Teams", International Economic Review, 32, 561-577.

McGUIRE, T. and M. RIORDAN (1995), "Incomplete Information and Optimal Market Structure: Public Purchases from Private Providers", Journal of Public Economics, 56, 125-141.

PICARD, P. et P. REY (1990), "Incentives in Cooperative Research and Development" In Essays of Edmond Malinvaud, vol 1, 252-279, MIT Press.

RIORDAN, M. (1994), "Contracting with Qualified Suppliers", BU. Discussion Paper.

SAPPINGTON, D. (1983), "Optimal Regulation of a Multiproduct Monopoly with Unknown Technological Capabilities" Bell Journal of Economics 14, 453-463.

SPIEWAK, S. and L. WEISS (1991), "The Wheeling and Transmission Manual", Fairmont Press, Inc.

TIROLE, J. (1988), "The Theory of Industrial Organisation", MIT Press.

WATKISS, J. and D. SMITH (1993), "The Energy Policy Act of 
1992-A Watershed for Competition in the Wholesale Power Market", Yale Journal on Regulation, 2, 447-492.

WOLINSKY, A. (1994), "Regulation of Duopoly: Managed Competition vs Regulated Monopolies", working paper Northwestern University. 


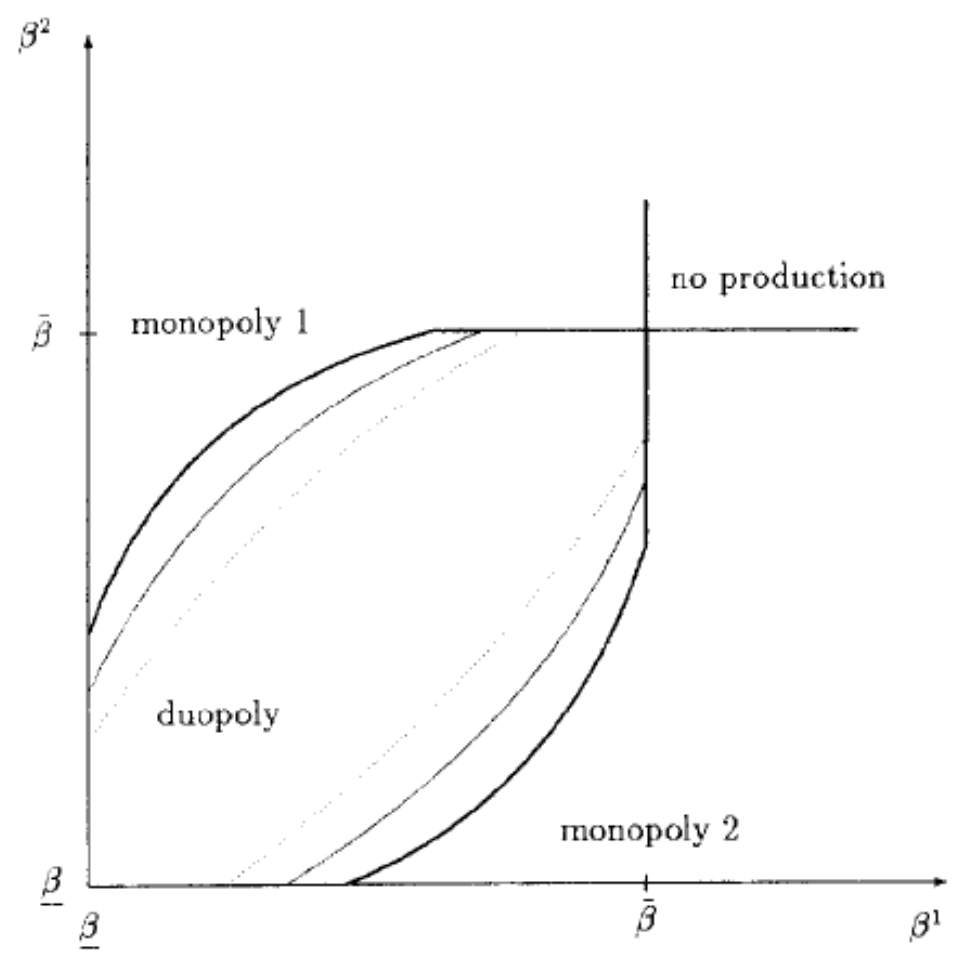

Fig. 1. Optimal market structure. — duopoly region under complete information; region under incomplete information, verifiable quality: $\cdots$ duspoly region under incomplete information, unverifiable quality. 